\title{
Surgical Approach Algorithm in the Treatment of Lumbar and Thoracolumbar Pyogenic Spondylodiscitis
}

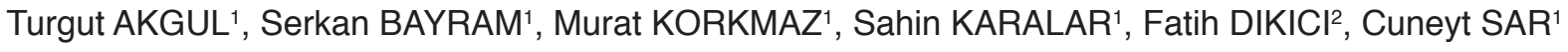 \\ ${ }^{1}$ Istanbul University, Istanbul Faculty of Medicine, Department of Orthopedics and Traumatology, Istanbul, Turkey \\ ${ }^{2}$ Acibadem Mehmet Ali Aydinlar University, Faculty of Medicine, Department of Orthopedics and Traumatology, Istanbul, Turkey \\ This study has been presented as an oral presentation at the $1^{\text {st }}$ Virtual National Orthopedic Congress, 2020.
}

Corresponding author: Serkan BAYRAM dr.serkanbayram89@gmail.com

\section{ABSTRACT}

\begin{abstract}
AIM: This study aimed to reveal the efficiency of our surgical approach algorithm in patients with thoracolumbar pyogenic spondylodiscitis based on the involvement of anatomical structure.

MATERIAL and METHODS: Data of patients who underwent debridement or stabilization surgery for thoracolumbar pyogenic spondylodiscitis from January 2012 to December 2018 were reviewed. Lumbar and thoracolumbar spondylodiscitis was classified into four stages based on anatomical involvement. Infection was limited in the disc space, which had not spread to the endplate in stage 1 and progressed as two-level corpus involvement of $>1 / 2$ of vertebral corpus bony destruction or as failed treatment in stage 4. Neurological function was evaluated using Frankel's grading postoperatively. Functional outcomes were categorized according to the Kirkaldy-Willis criteria.
\end{abstract}

RESULTS: The study included 39 patients, with a mean age of 58.2 years. Of these patients, 10, 12, 13, and 4 had stages 1, 2, 3 , and 4 spondylodiscitis, respectively. The mean follow-up period was 60.2 (12-184) months. All patients with stages 1 and 2 spondylodiscitis had grade E injury; 2 and 10 patients with stage 3 had grades $D$ and $E$ injuries, respectively; two patients with stage 4 had grade $D$ injury and two had grade $E$ injury at the last follow-up. Moreover, $100 \%, 84.6 \%$, and $50 \%$ of the patients with stages 1 and 2, 3, and 4 spondylodiscitis achieved good or excellent results, respectively.

CONCLUSION: The choice of the surgical technique depends on the destruction severity at the adjacent vertebral corpus. Surgical staging system for spondylodiscitis is useful and reliable in choosing appropriate surgical techniques.

KEYWORDS: Pyogenic spondylodiscitis, Surgical algorithm, Debridement, Functional status

ABBREVIATIONS: AP: Anteroposterior, CT: Computed tomography, MRI: Magnetic resonance imaging, TLIF: Transforaminal lumbar interbody fusion

\section{INTRODUCTION}

pondylodiscitis is a bacterial infection that affects the interis intervertebral disc and adjacent vertebral corpus. Its incidence was reported as 2.4 cases per 100,000 people, and this rate increases with age and immunosuppression. The morbidity rate varies from $5 \%$ to $56 \%$. In addition, untreated spondylodiscitis may result in neurological deficits and spinal deformity $(4,14,17)$.

In the literature, there is a consensus on diagnostic modalities $(2,4,17)$. However, there are many arguments on treatment
Turgut AKGUL (D) : 0000-0002-0704-3797

Serkan BAYRAM (D): 0000-0001-7651-1200

Murat KORKMAZ (1) : 0000-0003-2809-6721
Sahin KARALAR (10) : 0000-0002-4386-9107

Fatih DIKICI (1D): 0000-0003-2681-0098

Cuneyt SAR (1) : 0000-0002-5039-6280 
modalities, such as conservative or combination with surgery. Although there is no consensus on surgical treatment option or timing $(3,18)$ some studies recommend that external stabilization can provide satisfactory results compared with surgery even with the existence of neurological impairment $(8,16,23)$. Ultimately, surgical treatment was recommended in patients unresponsive to antibiotic treatment, with severe kyphotic deformity, progressive instability, epidural abscess, and neurological deficits $(1,3,16,18,23)$.

The gold standard for surgical treatment is debridement of the infected area and maintenance of stability. Achievement of stability is required in the treatment of osteomyelitis. In the literature, satisfactory results were reported with either minimally invasive procedure or open surgery. Unfortunately, most of these studies were case series. However, the reported cases were not categorized based on disease severity $(3,16,18,23)$. Some studies classified spondylodiscitis according to spreading infection, which was based on neurological status and instability $(2,3,8,16)$. In the light of knowledge presented in the literature with advanced surgical treatment, we believe that the surgical approach for the thoracic and lumbar areas should be considered separately due to anatomical restriction.

This study aimed to show the efficiency of our surgical approach algorithm in patients with pyogenic spondylodiscitis based on the involvement of the adjacent anatomical structure.

\section{MATERIAL and METHODS}

After the Institutional Review Board approved our study, we retrospectively reviewed the prospectively recorded data of all patients who underwent debridement or stabilization surgery for pyogenic spondylodiscitis from January 2012 to December 2018. This study included 90 patients with spondylodiscitis who underwent surgery in our clinic. The medical histories and radiographic images of the patients were assessed using the medical registration files. We evaluated the thoracic and lumbar spine separately.

Inclusion criteria: Patients diagnosed with pyogenic spondylodiscitis, with a minimum follow-up of 1 year, lumbar and thoracolumbar junction spondylodiscitis, and those with available demographics and medical records.

Exclusion criteria: Thoracic cases higher than T11, specific infection, such as tuberculosis and Brucella osteomyelitis, cervical spondylodiscitis, and patients treated conservatively.

Lumbar and thoracolumbar spondylodiscitis was classified into four stages based on anatomical involvement. Standard anteroposterior (AP) and lateral X-ray, computerized tomography (CT), and magnetic resonance imaging (MRI) were performed for all patients included in the study.

In patients diagnosed with spondylodiscitis whose clinical and radiological evaluations were available, antibiotherapy was started immediately after biopsy was taken under CT guidance or isolated with blood culture. If the patients have neurological deficit or epidural abscess, immediate surgical approach was performed. By contrast, in patients who had discitis alone without neurological deficit, antibiotherapy was started and bed rest was recommended. In this method, patients were mobilized with thoracolumbar orthosis for assistance. If pain was not controlled with medications or serum inflammatory markers were increased under antibiotic treatment, conservative treatment was considered unsuccessful.

All patients consulted the same infection specialist before and after surgery. All patients with stages 1 and 2 spondylodiscitis were treated conservatively, and aggressive antibiotherapy was administered at the previous infective disease clinic. This treatment method failed, and clinical status worsened in all patients at stages 1 and 2. In patients who developed sepsis, operative management was preferred for early local control of infection, especially in patient at stages 1 and 2 spondylodiscitis. However, patients with stages 3 and 4 spondylodiscitis underwent surgery because of instability rather than infection status. Specifically, the preoperative neurological status of patients with stage 4 spondylodiscitis was half Frankel $\mathrm{C}$ and half Frankel D. Clinical instability was defined by White and Panjabi as the inability of the spine to maintain its normal displacement under physiological loads, which does not result in neurological deficits, incapacitating deformity, or severe pain (25).

\section{Surgical Procedure}

All patients were prepared in the prone position on the fluoroscopy table. All surgeries were performed by two surgeons who specialized in spine surgery at a dual center.

Stage 1: In this stage, infection is limited in the disc space. Infection has not spread to the endplate through the adjacent vertebral corpus. In this stage, radiological examination shows disc space height loss and foraminal stenosis. T2-weighted MRI also showed edema at the epical area, and CT showed no defective area adjacent to the vertebral corpus. In this stage, all patients underwent surgery via only the posterior approach and transforaminal lumbar interbody fusion (TLIF) from the mostly affected foraminal side with hemilaminectomy. The other side of the lamina was protected to achieve bony fusion. Posterior stabilization was achieved using a pedicle screw (Figure 1).

Stage 2: In this stage, the infection spreads to the adjacent vertebral corpus from the affected disc space through the endplate. Radiological examination shows disc space height loss and foraminal stenosis without increase of kyphosis degree $<25^{\circ}$. T2-weighted MRI also showed edema of the entire adjacent vertebral corpus; $<1 / 3$ of vertebral corpus bony destruction does not pass the pedicle level at CT. Patients without and with instability were classified as stage $2 a$ and $2 \mathrm{~b}$, respectively. This stage is the transitional stage to choose the surgical approach. Patients classified as stage 2 could be treated similar to those in stage 1. Mega TLIF cage, with height of 14 to $18 \mathrm{~mm}$, was manufactured for this stage. The mega TLIF cage is a special product for this type of surgery and produced by a Turkish company. The maximum height of the TLIF cage is smaller than that of the anterior lumbar or direct lateral interbody fusion cage. At standard TLIF instrumentation set, the maximum cage height is $13 \mathrm{~mm}$. The 


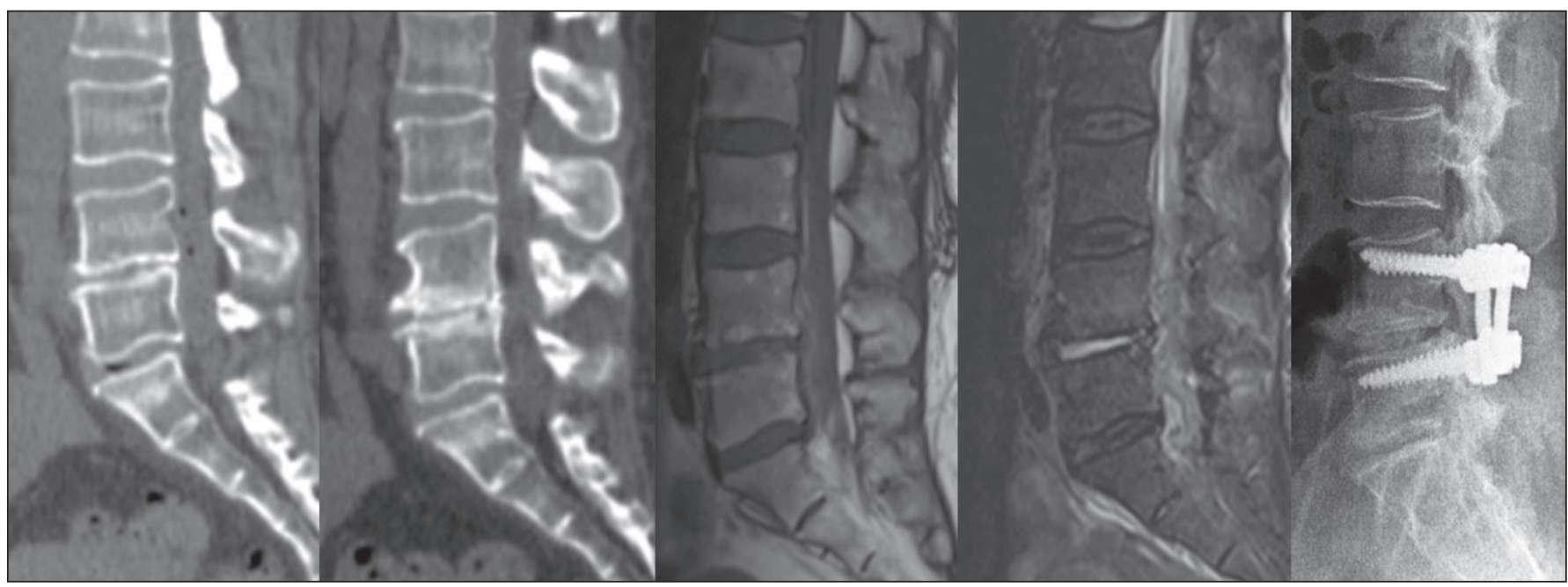

Figure 1: In stage 1, sagittal CT shows disc space narrowing without bony destruction. MRI shows T1- and T2-weighted disc space involvement. Postoperative lateral X-ray in a patient who underwent TLIF using iliac bone grafting shows increased disc space height.

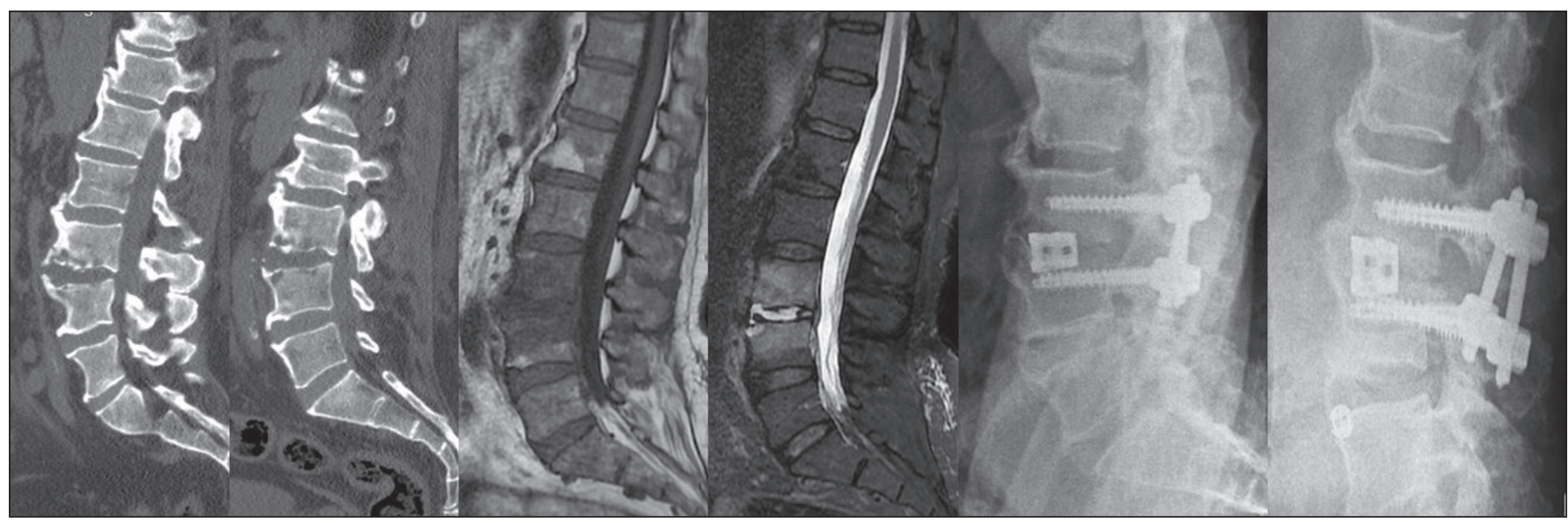

Figure 2: In stage 2A, sagittal CT shows peridiscal bony destruction wherein $<1 / 3$ of vertebral corpus bony destruction does not pass the pedicle level. MRI shows T1- and T2-weighted disc space involvement with extended bony edema of the entire corpus. Postoperative lateral X-ray in a patient who underwent TLIF using Mega titanium TLIF cage shows increased disc space height, and the 6-month control shows full union.

height of the mega TLIF cage ranges from $13 \mathrm{~mm}$ to $17 \mathrm{~mm}$, and it is inserted with the transforaminal technique (Figure 2, 3).

Stage 3: In this stage, the infection spreads to the adjacent vertebral corpus from the affected disc space through the endplate. Radiological examination shows disc space height loss and foraminal stenosis with increase of kyphosis degree $>25^{\circ}$. T2-weighted MRI also showed edema of the entire adjacent vertebral corpus, and CT showed that vertebral corpus bony destruction extended to the inferior vertebrae through the pedicle level. Patients with stage 3 underwent combined surgery. Anterior debridement with the anterior column was supported with viable strut graft after correction of segmental kyphosis. Posterior fusion was performed using a pedicle screw and rod combination (Figure 4).

Stage 4: In this stage, radiological examination showed two-level corpus involvement of $>1 / 2$ of vertebral corpus bony destruction or failed treatment requiring an extensive approach. Two-level corpectomy is necessary, which could be performed with an anterior approach. Structural allografts combined with titanium cage were used to provide anterior colon support. Posterior fusion using pedicle screw and rod combination were added to surgery (Figure 5, 6).

Intraoperative specimens from necrotic material were obtained for histopathological evaluation, detection of causative microorganism, and for culture and sensitivity testing in all cases. Finally, $2 \mathrm{~g}$ vancomycin, which was spread over the operative field after wound irrigation, was used as prophylaxis for surgical site infection.

\section{Postoperative Care and Follow-up}

Opioid and non-steroid anti-inflammatory drugs were administered for postoperative pain management. Immediately, postoperative AP and lateral views were routinely performed 


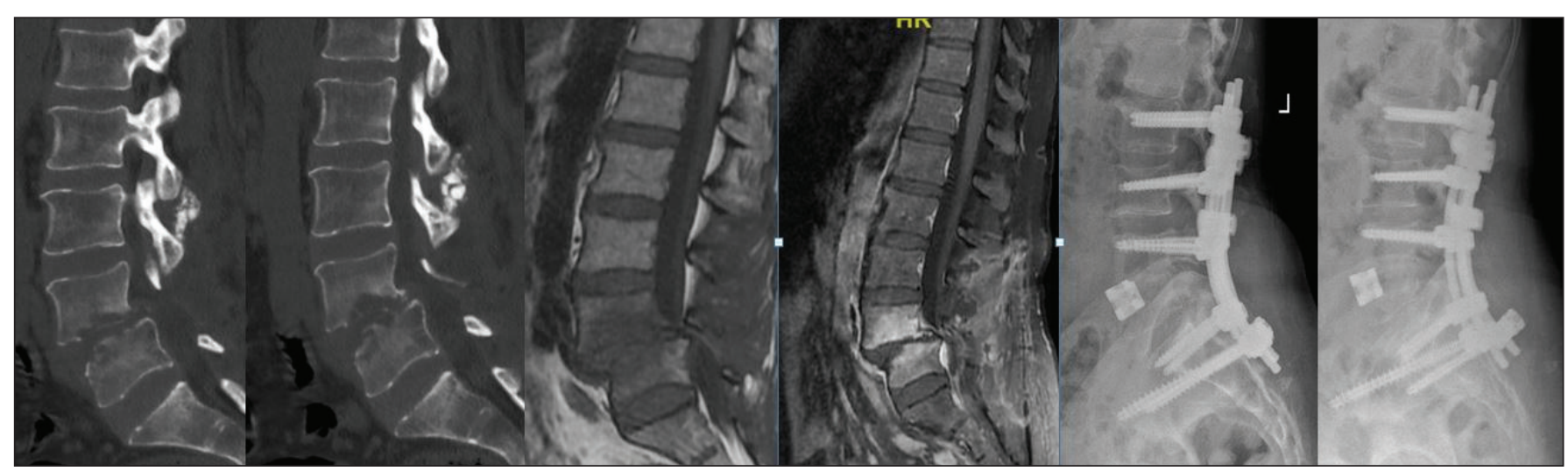

Figure 3: In stage 2B, sagittal CT shows peridiscal bony destruction and listhesis. MRI shows T1- and T2-weighted disc space involvement with extended bony edema of the entire corpus. Postoperative lateral X-ray in a patient who underwent TLIF using Mega titanium TLIF cage shows increased disc space height, and the 6-month control shows full union.

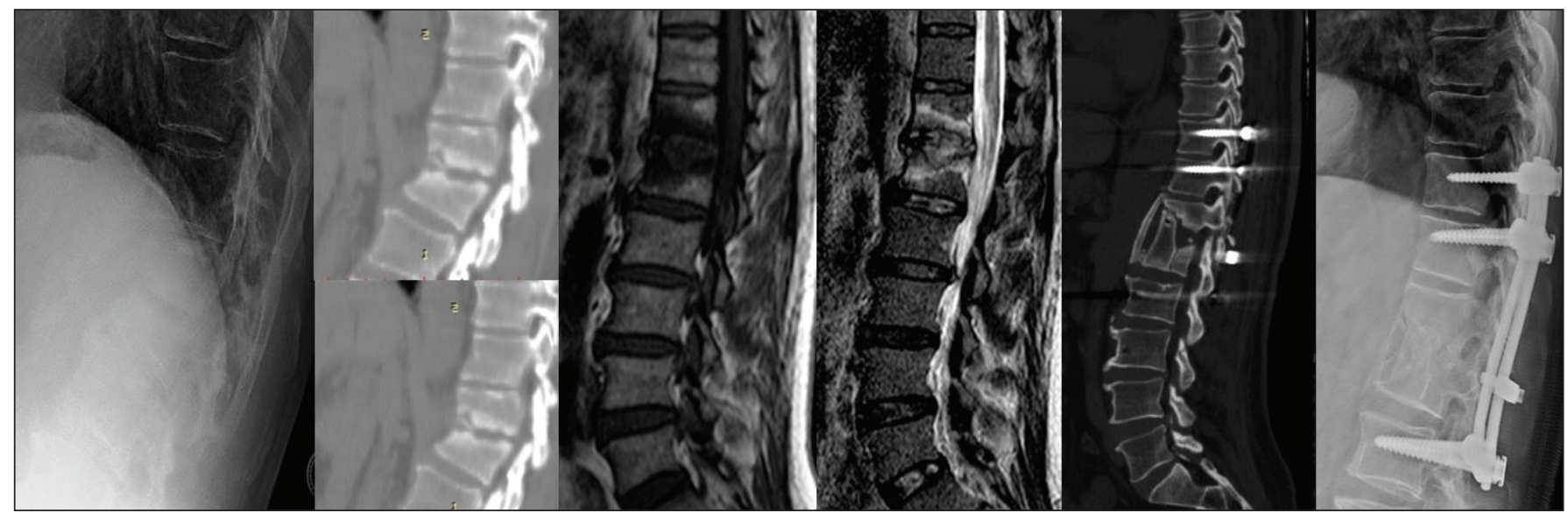

Figure 4: In stage 3, lateral X-ray shows segmental kyphosis of $>25^{\circ}$. CT shows vertebral corpus bony destruction extended to the inferior vertebrae through the pedicle level. MRI shows T1- and T2 weighted disc space and bony destruction. Early postoperative sagittal CT view shows segmental kyphosis correction with combined approach anterior debridement and iliac bone graft insertion posterior pedicle screw rod combination. One-year follow-up lateral X-ray shows fusion.

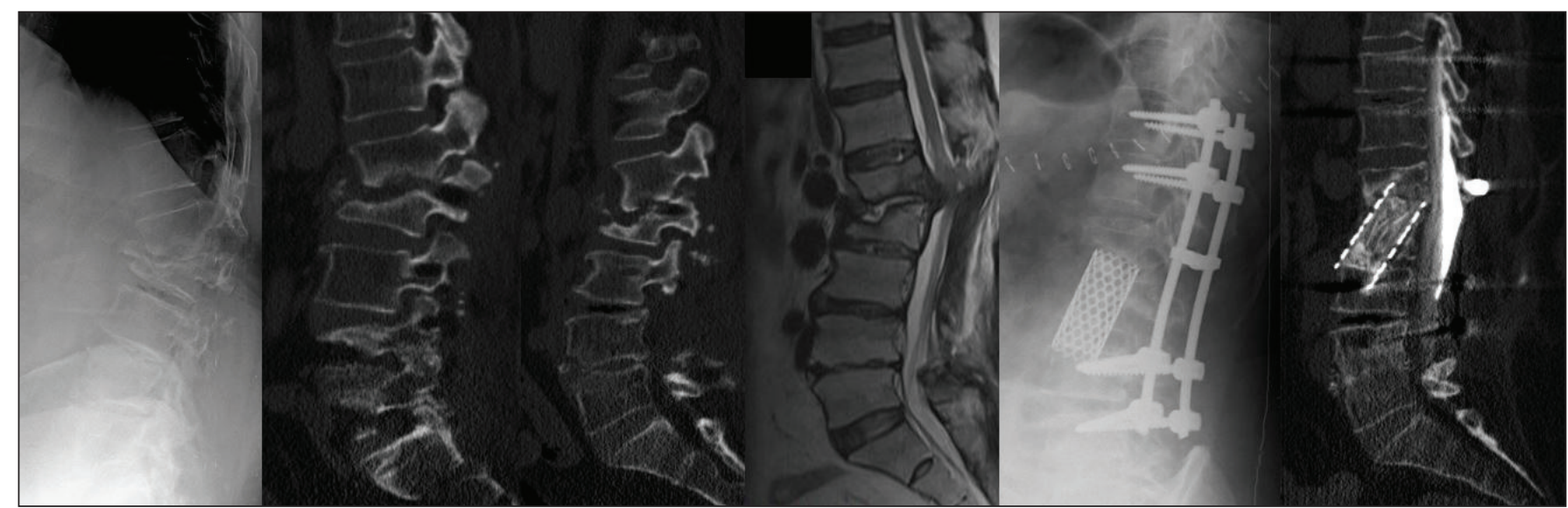

Figure 5: In stage 4, lateral X-ray shows two-level corpus involvement of $>1 / 2$ of vertebral corpus bony destruction. Sagittal CT view, T1-weighted sagittal MRI view also showed the destruction. Early postoperative lateral X-ray view shows segmental kyphosis correction with combined approach anterior debridement and titanium cages filled with iliac bony graft insertion posterior pedicle screw rod combination. One-year follow-up sagittal CT shows fusion with minimally decreased lordosis angle. 


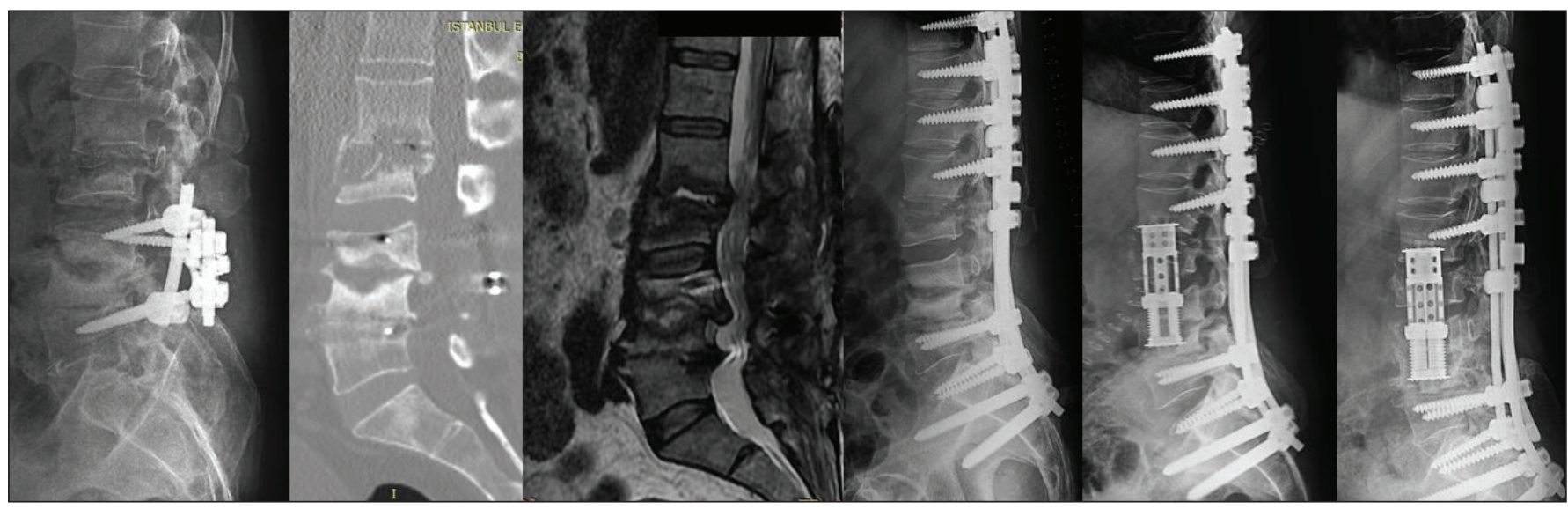

Figure 6: In stage 4, radiological examination shows two-level corpus involvement of $>1 / 2$ of vertebral corpus bony destruction after failed surgical intervention. Lateral X-ray shows three-vertebra corpus involvement. Sagittal CT and MRI show bond destruction and disc space involvement. The patient underwent staged combined approach due to medical condition. Lateral X-ray was taken immediately after the first surgery for posterior stabilization. In the second stage, anterior debridement was performed, and fibula strut graft combined with expandable titanium cage was used. At 6-months follow-up, lateral X-ray shows good autogenous strut fibula graft with maintenance of good sagittal plane correction.

for all patients to evaluate implant or graft placement, sagittal and coronal alignment, and deformity correction. Standard parenteral antibiotic (generally third-generation cephalosporins) was started postoperatively and continued until drain removal ( 1 or 2 days postoperatively). Once microbiological evaluation was completed, and if a microorganism was detected, specific antibiotherapy was started and continued for 2-4 weeks intravenously, and oral antibiotics were continued for 3 months according to the culture and sensitivity results with strict monitoring of laboratory investigations (e.g., total blood counts, erythrocyte sedimentation rate, and C-reactive protein [CRP]).

Patients were rehabilitated immediately based on their tolerance level. All patients were permitted to sit on the bed or walk supported with a thoracolumbosacral orthosis, which was continued for 3 months with a walker. Passive and active movements of the trunk and lower limb muscles were individualized after discharge.

Subsequently, the patients were followed up at the outpatient clinic for 1, 3, 6 and 12 weeks, 6 months, 1 year, and thereafter, once a year.

\section{Outcome Measures}

The neurological function of all patents was evaluated using Frankel grading pre- and postoperatively. Frankel grade for spinal cord injury: A: Complete loss of motor and sensory function; B: incomplete, preserved sensation only; C: incomplete, preserved motor (non-functional); D: incomplete, preserved motor (functional); E: complete return of all motor and sensory functions but may have abnormal reflexes (5).

Functional outcomes in all patients were categorized as excellent, good, fair, and poor according to the Kirkaldy-Willis criteria (11).

\section{RESULTS}

The study included 39 patients (18 women and 21 men), with a mean age of $58.2 \pm 8.4$ years (range, $47-72$ years). Of these patients, 10, 12, 13, and 4 patients had stage 1, 2, 3, and 4 spondylodiscitis, respectively (Table I). The mean follow-up period was 60.2 months (12-184).

\section{Neurological Results}

In stage 1, one patient had Frankel grade D injury and nine had grade E injury preoperatively; at the last follow-up, all patients had grade $E$ injury.

In stage 2, four patients had grade $C$ injury, four had grade $D$ injury, and four patients had grade $\mathrm{E}$ injury postoperatively; at the last follow-up, all patients had grade E injury.

In stage 3, two patients had grade B injury, five had grade $C$ injury, four had grade $D$ injury, and two had grade $E$ injury preoperatively; at the last follow-up, three and ten patients had grade $D$ and $E$ injuries, respectively.

In stage 4, two patients had grade $\mathrm{C}$ injury, and two had grade D injury preoperatively; at the last follow-up, two patients had grade D injury, and two had grade $E$ injury.

Functional evaluation at the final visit showed excellent results in eight patients and good in two patients in stage 1, with $100 \%$ of patients achieving a good or excellent result. In stage 2 , excellent and good results were achieved in eight and four patients, respectively, with $100 \%$ of patients having a good or excellent result. In stage 3, excellent, good, and fair results were achieved in four, seven, and two patients, respectively, with $84.6 \%$ of patients having a good or excellent result. In stage 4 , good and fair results were achieved in two and two patients, respectively, with $50 \%$ of patients having a good result.

The mean preoperative erythrocyte sedimentation rate (ESR) and CRP level were $86 \pm 16 \mathrm{~mm} / \mathrm{h}$ and $77.2 \pm 33 \mathrm{mg} / \mathrm{L}$ in stage 
Table I: Flow Diagram of Patients and Categorization of Study Groups

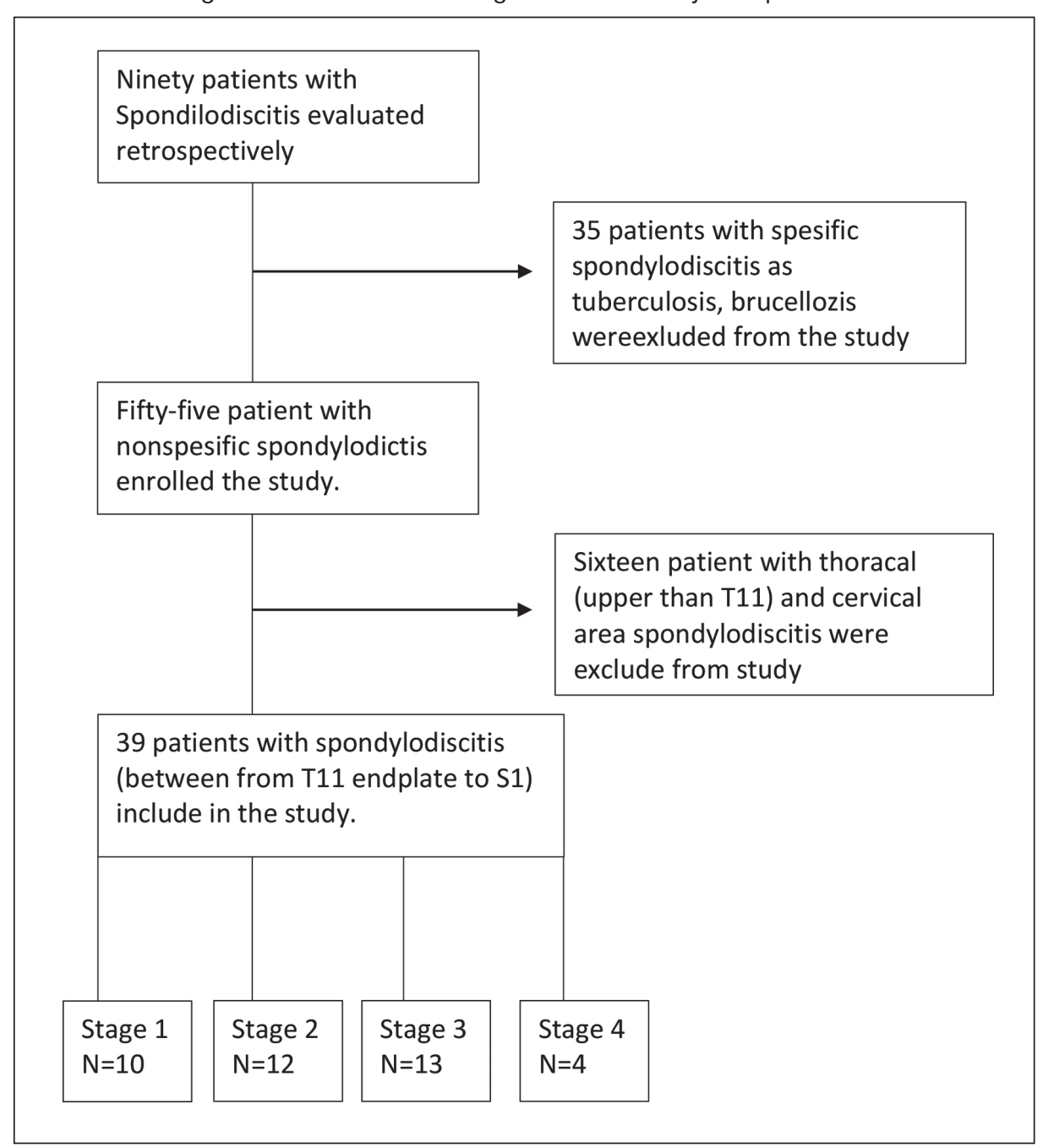

$1,73.8 \pm 13 \mathrm{~mm} / \mathrm{h}$ and $61 \pm 29 \mathrm{mg} / \mathrm{L}$ in stage $2,44 \pm 10 \mathrm{~mm} / \mathrm{h}$ and $20 \pm 13 \mathrm{mg} / \mathrm{L}$ in stage 3 , and $33.2 \pm 8 \mathrm{~mm} / \mathrm{h}$ and $17 \pm 9$ $\mathrm{mg} / \mathrm{L}$ in stage 4. All ESR and CRP levels decreased to normal limits at the final follow-up.

No major complications were observed after these procedures. No additional surgery was required for any patient. In one patient, a vacuum-assisted closure system was used for prolonged drainage without an irrigation system. By contrast, the drain was removed from the patients when the total drainage amount was $<50 \mathrm{cc} /$ day.

\section{DISCUSSION}

The incidence of spondylodiscitis increases with aging and immunosuppression due to chronic disease. Spine surgeons and infection disease specialists can treat this disease as a team. In the literature, there is an agreement on the diagnostic tools and criteria. Long-term antibiotic treatment is recommended after isolating the bacterial agent in culture (2). By contrast, surgical intervention is recommended in patients with progressive neurological deficits, instability, and failed conservative treatment.

There is no consensus on surgical treatment of early-stage spondylodiscitis. Bettini et al. reported good results with only external stabilization and antibiotherapy combination without surgical treatment (3). Although conservative treatment of discitis without debridement is useful in infection eradication, we realized that the time course of antibiotic treatment is longer, and mobile lumbar spine is healed with residual deformity affecting other parts of the mobile lumbar spine (7). Particularly, space narrowing resulted in foraminal stenosis and segmental kyphosis, which affect patient satisfaction (6). The complication rates ranged from $12 \%$ to $18 \%(3,22)$. Early surgeries providing rapid recovery without disability were recommended by some studies as case series $(10,21)$. Therefore, we categorized early discitis as stage 1 nonspecific spinal pain, and surgery is suggested for incredible pain that negatively affect mobilization.

Rutges et al. reviewed 25 studies focusing on spondylodiscitis. Consequently, they reported that isolated anterior approach 
could result in better clinical outcome (18). Many techniques, such as minimally invasive, anterior, and posterior combined procedures were introduced with high success, with relapse rates ranging from $0 \%$ to $10 \%$. Debridement of the infected tissue until viable tissue is reached has been known to be the key factor that determines surgical treatment success. Comparative studies showed that both surgical approaches have satisfactory results in debridement of infected tissue $(23,26)$. Stabilization could be performed via posterior approach open or percutaneously either via anterior approach. İsolated anterior debridement and fusion have superior results than combination due to the single approach $(3,13,24)$. We believe that anterior support is mandatory in maintaining stability and achieving solid fusion. Recently, technology improvement offers an option to provide anterior support through only the posterior approach in selective cases $(8,9,15)$. On the contrary, these cases reported in literature did not have a uniform involvement. Spondylodiscitis varies from basic discitis to spinal instability with huge kyphosis angle. Therefore, providing the same treatment options is impossible for every case with varied severity.

The literature lacks a surgical algorithm for treatment choice due to difficulty in classification. The PS classification presented by Akbar et al. based on neurological status and instability gives details for general treatment options and has a lack of surgical option choice (1). Similarly, Pola et al. presented their classification system based on similar criteria, and the algorithm is almost the same in a previous study (16). Recently, Homagk et al. introduced spondylodiscitis severity scores and classified them into three main groups $(8,20)$. This classification provides surgeons a preference for their treatment choice based on the criteria for thoracic and lumbar spondylodiscitis. As reported and supported by our study, treatment should be chosen based on classification. The present classification based on anatomical involvement of bone destruction is useful in choosing the surgical approach with high success rate.

We recommend not only one approach for all stages of spondylodiscitis. The early stage of the infection could be treated with only the posterior approach by using TLIF (24). Shetty et al. also reported satisfactory results with TLIF in the treatment of spondylodiscitis (20). We believe that posterior access has a limitation due to nerve structure position that restrains in supporting the anterior column with higher titanium cage filled with autogenous or strut graft. According to our staging, we recommend the combined approach to correct patients with increased kyphotic deformity, and debridement is necessary if infection involves more than one vertebral corpus. We also suggest that using titanium cage is safe in supporting the anterior colon in the treatment of discitis. Korovessis et al. and Schomacher et al. reported that using a titanium cage is safe and reliable tool even in an infected area after adequate debridement $(12,19)$.

This study has several limitations. This is a retrospective study with a relatively small number of cases. Patient-specific covariates such as smoking habit and medical comorbidities were not evaluated, which affected the union rate and functional results. In this study, there was no control group for comparison of the effectiveness of the algorithm.

In the present study, debridement and stabilization in surgical treatment of pyogenic spondylodiscitis have satisfactory results. Surgical technique choice depends on the destruction severity at the adjacent vertebral corpus. Surgical staging system for spondylodiscitis is useful and reliable in choosing appropriate surgical techniques.

\section{REFERENCES}

1. Akbar $\mathrm{M}$, Lehner $\mathrm{B}$, Doustdar $\mathrm{S}$, Fürstenberg $\mathrm{CH}$, Hemmer S, Bruckner T, Carstens C, Wiedenhöfer B: Pyogenic spondylodiscitis of the thoracic and lumbar spine: A new classification and guide for surgical decision-making. Orthopade 40(7):614-623, 2011

2. Appalanaidu N, Shafafy R, Gee C, Brogan K, Karmani S, Morassi G, Elsayed S: Predicting the need for surgical intervention in patients with spondylodiscitis: The Brighton Spondylodiscitis Score (BSDS). Eur Spine J 28(4):751-761, 2019

3. Bettini N, Girardo M, Dema E, Cervellati S: Evaluation of conservative treatment of non specific spondylodiscitis. Eur Spine J 18 Suppl 1:143-150, 2009

4. Cheung WY, Luk KD: Pyogenic spondylitis. Int Orthop 36(2):397-404, 2012

5. Frankel HL, Hancock DO, Hyslop G, Melzak J, Michaelis LS, Ungar GH, Vernon JD, Walsh JJ: The value of postural reduction in the initial management of closed injuries of the spine with paraplegia and tetraplegia. I. Paraplegia 7:179-192, 1969

6. Gasbarrini AL, Bertoldi E, Mazzetti M, Fini L, Terzi S, Gonella F, Mirabile L, Barbanti Bròdano G, Furno A, Gasbarrini A, Boriani $\mathrm{S}$ : Clinical features, diagnostic and therapeutic approaches to haematogenous vertebral osteomyelitis. Eur Rev Med Pharmacol Sci 9(1):53-66, 2005

7. Hadjipavlou AG, Mader JT, Necessary JT, Muffoletto AJ: Hematogenous pyogenic spinal infections and their surgical management. Spine (Phila Pa 1976) 25(13):1668-1679, 2000

8. Homagk L, Homagk N, Klauss JR, Roehl K, Hofmann GO, Marmelstein D: Spondylodiscitis severity code: Scoring system for the classification and treatment of non-specific spondylodiscitis. Eur Spine J 25(4):1012-1020, 2016

9. Homagk L, Marmelstein D, Homagk N, Hofmann GO: SponDT (Spondylodiscitis Diagnosis and Treatment): Spondylodiscitis scoring system. J Orthop Surg Res 14(1):100, 2019

10. Kamal AM, El-Sharkawi MM, El-Sabrout M, Hassan MG: Spondylodiscitis: Experience of surgical management of complicated cases after failed antibiotic treatment. SICOT J 6:5, 2020

11. Kirkaldy-Willis WH, Paine KW, Cauchoix J, Mclvor G: Lumbar spinal stenosis. Clin Orthop Relat Res 99:30-50,1974

12. Korovessis P, Repantis T, lliopoulos P, Hadjipavlou A: Beneficial influence of titanium mesh cage on infection healing and spinal reconstruction in hematogenous septic spondylitis: A retrospective analysis of surgical outcome of twenty-five consecutive cases and review of literature. Spine (Phila Pa 1976) 33(21):E759-E767, 2008 
13. Lee JS, Suh KT: Posterior lumbar interbody fusion with an autogenous iliac crest bone graft in the treatment of pyogenic spondylodiscitis. J Bone Joint Surg Br 88(6):765-770, 2006

14. Li YD, Wong CB, Tsai TT, Lai PL, Niu CC, Chen LH, Fu TS: Appropriate duration of post-surgical intravenous antibiotic therapy for pyogenic spondylodiscitis. BMC Infect Dis 18(1):468, 2018

15. Lin CP, Ma HL, Wang ST, Liu CL, Yu WK, Chang MC: Surgical results of long posterior fixation with short fusion in the treatment of pyogenic spondylodiscitis of the thoracic and lumbar spine: A retrospective study. Spine (Phila Pa 1976) 37(25): E1572-E1579, 2012

16. Pola E, Autore G, Formica VM, Pambianco V, Colangelo D, Cauda R, Fantoni M: New classification for the treatment of pyogenic spondylodiscitis: Validation study on a population of 250 patients with a follow-up of 2 years. Eur Spine J 26 Suppl 4:479-488, 2017

17. Quack V, Hermann I, Rath B, Dietrich K, Spreckelsen C, Lüring C, Arbab D, Mueller CA, Shousha M, Clusmann H, Tingart M: Current treatment strategies for spondylodiscitis in surgical clinics in Germany. Z Orthop Unfall 152(6):577-583, 2014

18. Rutges JP, Kempen DH, van Dijk M, Oner FC: Outcome of conservative and surgical treatment of pyogenic spondylodiscitis: A systematic literature review. Eur Spine J 25(4):983-999, 2016

19. Schomacher M, Finger T, Koeppen D, Süss O, Vajkoczy P, Kroppenstedt S, Cabraja M: Application of titanium and polyetheretherketone cages in the treatment of pyogenic spondylodiscitis. Clin Neurol Neurosurg 127:65-70, 2014
20. Shetty AP, Aiyer SN, Kanna RM, Maheswaran A, Rajasekaran S: Pyogenic lumbar spondylodiscitis treated with transforaminal lumbar interbody fusion: Safety and outcomes. Int Orthop 40(6):1163-1170, 2016

21. Tsai TT, Yang SC, Niu CC, Lai PL, Lee MH, Chen LH, Chen WJ: Early surgery with antibiotics treatment had better clinical outcomes than antibiotics treatment alone in patients with pyogenic spondylodiscitis: A retrospective cohort study. BMC Musculoskelet Disord 18(1):175, 2017

22. Valancius K, Hansen ES, Høy K, Helmig P, Niedermann $B$, Bünger $C$ : Failure modes in conservative and surgical management of infectious spondylodiscitis. Eur Spine $\mathrm{J}$ 22(8):1837-1844, 2013

23. Včelák J, Chomiak J, Toth L: Surgical treatment of lumbar spondylodiscitis: A comparison of two methods. Int Orthop 38(7):1425-1434, 2014

24. Vcelák J, Tóth L: Surgical treatment of spondylodiscitis. Acta Chir Orthop Traumatol Cech 75(2):110-116, 2008

25. White AA 3rd, Johnson RM, Panjabi MM, Southwick WO: Biomechanical analysis of clinical stability in the cervical spine. Clin Orthop Relat Res 109:85-96, 1975

26. Yaldiz C, Yaldiz M, Ceylan N, Kacira OK, Ceylan D, Kacira T, Kizilcay G, Tanriverdi T: A retrospective study of 39 patients treated with anterior approach of thoracic and lumbar spondylodiscitis: Clinical manifestations, anterior surgical treatment, and outcome. Medicine (Baltimore) 94(29):e1177, 2015 